\title{
Etnobotânica nordestina: estudo comparativo da relação entre comunidades e vegetação na Zona do Litoral - Mata do Estado de Pernambuco, Brasil
}

\author{
Alberto Jorge da Rocha Silva ${ }^{1,2}$ e Laise de Holanda Cavalcanti Andrade ${ }^{1}$
}

Recebido em 23/05/2003. Aceito em 13/07/2004

\begin{abstract}
RESUMO - (Etnobotânica nordestina: estudo comparativo da relação entre comunidades e vegetação na Zona do Litoral - Mata do Estado de Pernambuco, Brasil). O trabalho visa determinar as relações entre comunidades e vegetação na Zona da Mata de Pernambuco. A comunidade da Usina São José (Igarassu - Ig) é formada por vilas rurais, vizinhas da Reserva Ecológica da Mata da Usina São José. Jaguarana (Paulista-Pa) é uma comunidade urbana, próxima à Reserva Ecológica da Mata de Jaguarana. Levantou-se as plantas úteis para estas comunidades por meio de 38 entrevistas não-estruturadas, enquadrando-as nas categorias de uso alimentação, comércio, construção, mágico, medicinal, tecnológico e outros. Foram registradas 334 espécies, nativas e cultivadas. Comparou-se as comunidades, através do Quociente de Similaridade de Sørensen (Qs), obtendo-se maiores índices para plantas alimentícias $\left(\mathrm{Qs}_{\mathrm{IgPa}}=0,71\right)$, predominantemente cultivadas, e medicinais $\left(\mathrm{Qs}_{\mathrm{IgPa}}=0,56\right), 45,1 \%$ delas nativas, predominantemente herbáceas. A vegetação nativa constitui uma fonte importante de recursos medicinais mas é subutilizada como fonte de alimento e tem pouca relevância na visão cosmológica das comunidades. Os vegetais não têm relevância na atividade de comércio, servindo como complemento de renda para as famílias $\left(\mathrm{Qs}_{\mathrm{IgPa}}=0,30\right)$. Na categoria construção enquadram-se, majoritariamente, espécies nativas $(87,8 \%)$ e arbóreas $(92,7 \%)$. Em tecnologia têm maior representatividade espécies nativas $(85,7 \%)$ e arbóreo/arbustivas $(84,1 \%)$. As comunidades utilizam diferentes fontes vegetais para as categorias construção, mágica, tecnologia e outros, decorrentes tanto das principais atividades que nelas exercem seus moradores, como dos recursos naturais à sua disposição.
\end{abstract}

Palavras-chave: Etnobotânica, plantas úteis, categorias de uso, Mata Atlântica

\begin{abstract}
Northeast Ethnobotany: links between communities and vegetation of the Coast Zone of the Mata Region in Pernambuco State, Brazil). The objective of this work is to determine the social behaviour concerning the consumption of vegetal species by local communities. The involved localities are: the Usina São José (Igarassu - Ig), which involves rural communities in the surroundings of the Ecological Reserve of the Usina São José and the Jaguarana (Paulista - PE), an urban settlement located in the neighbourhoods of the Ecological Reserve of Jaguarana. Structured interviews were applied to these social groups, addressing 38 people, in order to identify the most useful plants and the correspondent use. These were classified into categories food, commerce, constrution, ritualistic, medicinal, technological and "others". Among these types, 334 native and introduced species were registered. The different communities were analysed and compared through the Sørensen Similarity Quotient (Qs). The higher incidence is of feeding plants $\left(\mathrm{Qs}_{\mathrm{IgPa}}=0.71\right)$, predominantly cropped and medical plants $\left(\mathrm{Qs}_{\mathrm{IgPa}}=0.56\right)$, from which $45.1 \%$ are natives and herbaceous. The native vegetation constitutes an important source of medicines. However, it has no relevance as food within the cosmological view of the community. Vegetables are irrelevant as timber in the trade sector, for income generation $\left(\mathrm{Qs}_{\mathrm{IgPa}}=0.30\right)$. For building proposals most species are native $(87.8 \%)$ and tree $(92.7 \%)$. For technological purpose the native species $(85.7 \%)$ and the tree/shrub $(84.1 \%)$, are the most representative category. The communities use different types of vegetal resource for the construction, ritualistic and technology among other categories, as a result of their main activities and of the available natural resources.
\end{abstract}

Key words: Ethnobotany, useful plants, categories of use, Atlantic Rain Forest

\section{Introdução}

As florestas tropicais vêm sofrendo, paulatinamente, uma redução de suas áreas, por meio de desmatamentos para retirada de madeira, exploração de recursos minerais, implantação de projetos agropecuários e queimadas criminosas. A simples implantação de Unidades de Conservação nas áreas de cobertura vegetal nativa remanescente não tem sido eficiente na proteção e recuperação desses ecossistemas extremamente ameaçados. É necessária a inclusão de projetos de manejo nessas áreas para que a própria sociedade, e não apenas o poder público, seja responsável pela conservação das mesmas. A

\footnotetext{
${ }^{1}$ Universidade Federal de Pernambuco, Departamento de Botânica, Laboratório de Etnobotânica e Botânica Aplicada (LEBA), CCB, Cidade Universitária, CEP 50670-901, Recife, PE, Brasil

2 Autor para correspondência: albertorocha@hotmail.com
} 
Etnobotânica vem realizando pesquisas com comunidades assentadas nas regiões de florestas tropicais, desenvolvendo instrumentos para avaliar os recursos vegetais utilizados nestas áreas e apontando propostas de uso sustentado das mesmas, como forma de conservar e recuperar esses ecossistemas (Anderson \& Posey 1985; Prance et al. 1987; PinedoVasquez et al. 1990; Kainer \& Duryea 1992; Phillips et al. 1994; Albuquerque 1999).

A Mata Atlântica é considerada a floresta tropical mais ameaçada do planeta, possuindo atualmente apenas $5,0 \%$ da sua cobertura original. Na Região Nordeste, a situação é ainda mais grave, sendo encontrados apenas fragmentos de mata, a maioria com menos de 1.000ha, o que pode levar rapidamente ao seu desaparecimento; essa devastação deve-se à super exploração dos recursos madeireiros, ao crescimento desenfreado da urbanização e, principalmente, à implantação e crescimento da agricultura canavieira, desde a época colonial (Melo 1978; Costa 1982; Andrade 1988). Poucos estudos têm sido realizados na região nordestina com o objetivo de desenvolver programas de conservação e recuperação da Mata Atlântica, inclusive em relação ao conhecimento e usos que as populações assentadas fazem dos recursos ainda encontrados nos remanescentes florestais (Victor \& Andrade 1991; Voeks 1996).

Este trabalho teve como objetivo realizar o levantamento das espécies úteis em duas comunidades localizadas na Zona do Litoral-Mata do Estado de Pernambuco, comparando os recursos utilizados em cada uma delas e estabelecendo as relações das mesmas com a vegetação nativa da região. Os resultados obtidos podem fornecer dados valiosos para a conservação do ecossistema envolvido, através do manejo e uso sustentado de seus recursos, além de servir de base para novos estudos etnobotânicos, a serem realizados em comunidades situadas na região da Mata Atlântica.

\section{Material e métodos}

Áreas e comunidades de estudo - Foram selecionadas para o estudo comunidades situadas na Região Metropolitana do Recife (RMR), Zona do Litoral-Mata de Pernambuco, por situarem-se próximas a Unidades de Conservação (UC).

No Município de Igarassu, com acesso pela PE-41, estão localizadas as comunidades de Engenho Piedade, Engenho D'água e Maria Feia, todas vizinhas da
Reserva Ecológica da Mata da Usina São José $\left(07^{\circ} 50^{\prime} \mathrm{S}, 35^{\circ} 00^{\prime} \mathrm{W}\right.$ Gr.), sendo formadas por vilas rurais e por diversos sítios pequenos, situadas próximas à Usina São José, onde habitam famílias que trabalham na produção canavieira, no campo e na indústria. Atualmente, elas vêm passando por um processo de modificação, com a retirada dos antigos moradores, por questões trabalhistas com o Grupo Petribom, proprietário da usina, e a implantação de uma nova vila para os atuais funcionários da empresa.

A Mata da Usina São José, localizada junto às comunidades, ocupa uma área de 323,3ha, sendo considerada um remanescente de Mata Atlântica. Foi definida como Reserva Ecológica da Mata da Usina São José pela Lei Estadual n. 9989, de 1987, que reconhece como Reservas Ecológicas as matas de preservação permanente da RMR e dispõe sobre os procedimentos básicos relativos à sua preservação. Foi incluída na lei por ser protetora do relevo e do solo, e protetora do sistema hidrográfico, no caso a bacia do Rio Botafogo (FIDEM 1987).

A comunidade de Jaguarana $\left(08^{\circ} 00^{\prime} \mathrm{S}, 34^{\circ} 55^{\prime} \mathrm{W}\right.$ Gr) é uma comunidade urbana isolada, situada no Município do Paulista, próxima à PE-22. Ela foi formada há cerca de 50 anos por trabalhadores das fábricas de tecido do Grupo Lundgren, que se instalaram junto à pequena localidade no seio da mata, chamada Uruba. Há cerca de 30 anos, trabalhadores de uma indústria de cal, localizada no mesmo município foram indenizados com lotes de terra junto à área citada, após o fechamento da mesma. Com a venda de parte desses lotes, novos moradores passaram a fazer parte da comunidade.

A mata de Jaguarana encontra-se situada ao Norte da comunidade, entre as PE-15 e PE-22, e os Rios Timbó e Paratibe, sendo que o acesso a ela é feito por qualquer dessas vias. É considerada um remanescente da Mata Atlântica, ocupando uma área de 332,8ha. Foi definida como Reserva Ecológica da Mata de Jaguarana pela Lei n. 9.989/87, tendo sido criada pelos critérios de proteção do relevo e do solo e protetora da qualidade ambiental urbana. Pela sua proximidade com os centros urbanos do município, a Mata de Jaguarana tem sido objeto de desmatamento, não se apresentando em bom estado de conservação (FIDEM 1987b).

Informações sócio-econômicas e etnobotânicas - As comunidades em estudo foram caracterizadas através de dados obtidos a partir de um questionário sóciocultural, aplicado nas residências. Os 38 informantes 
que participaram das entrevistas foram selecionados a partir dos dados levantados nos questionários, pelos critérios de tempo de residência na comunidade (mínimo 35 anos), idade (45-73 anos) e atividade profissional.

O levantamento das espécies utilizadas pelas comunidades foi realizado por meio de entrevistas nãoestruturadas, inquirindo os informantes sobre as plantas úteis, seus diversos fins e formas de uso nas comunidades em estudo. As espécies foram enquadradas, segundo a sua utilidade, nas seguintes categorias de uso: alimentação, comércio, construção, medicinal, tecnológico e outros (Prance et al. 1987), reconhecendo-se ainda a categoria mágica (Balée 1986). Após isso, determinaram-se as categorias que apresentaram um maior índice de ocorrência em cada comunidade, e em seguida relacionou-se estas categorias com seus perfis. Os resultados obtidos sobre as categorias de uso das plantas foram utilizados para comparar as duas comunidades, visando estabelecer as características próprias de cada uma e suas semelhanças, aplicando-se o Quociente de Similaridade de Sørensen (Sørensen 1978).

Com relação ao aspecto botânico, foram considerados o porte das plantas (arbóreo, arbustivo, herbáceo e lianas) e os modos de obtenção, enquadrando como cultivadas aquelas espécies que a comunidade cultiva em suas roças, sítios, hortas, quintais e jardins; nativas as que são obtidas pela comunidade na vegetação local; introduzidas as exóticas não manejadas, obtidas nas vizinhanças das residências ou mesmo nas UCs e compradas, aquelas plantas adquiridas no comércio local ou fora da comunidade.

As plantas indicadas em cada comunidade foram coletadas e herborizadas, sendo realizada a identificação taxonômica de cada espécie, pelos autores e por especialistas nacionais, entre eles Ana Maria du Bocage Neta, Fernando Antônio Távora Gallindo, Rita de Cássia Araújo Pereira e Valdelice Correia Lima, da Seção de Botânica e Ecologia, da Empresa Pernambucana de Pesquisa Agropecuária IPA. As plantas adquiridas pela comunidade através da compra não foram coletadas e herborizadas, por não serem encontradas nas áreas estudadas, o mesmo acontecendo com espécies cultivadas muito difundidas, identificadas pela literatura especializada. Além disso, não foi possível a coleta de umas poucas espécies por não serem mais encontradas nas comunidades. As espécies coletadas com material fértil foram incorporadas ao acervo do Herbário IPA — Dárdano de Andrade Lima (Holmgren et al. 1990).

\section{Resultados e discussão}

Os 21 informantes das comunidades da Usina São José, em Igarassu (Ig), indicaram os usos para 285 espécies. Em Jaguarana, Paulista $(\mathrm{Pa})$, os 17 entrevistados citaram 214 espécies. Considerando que várias espécies são de uso comum nas duas áreas, foram registradas 334 espécies úteis no total. Estas espécies pertencem a 72 famílias de dicotiledôneas e 14 de monocotiledôneas, além de quatro famílias de pteridófitas, destacando-se pelo número as Leguminosae $($ Caesalpinioideae $=12 ;$ Mimosoideae $=$ 15; Papilionoideae $=14)$, Lamiaceae (15), Euphorbiaceae (13), Myrtaceae (11) e Poaceae (10). Das seis famílias destacadas por Hanazaki et al. (2000) para duas comunidades caiçaras em São Paulo, pelo número de espécies úteis, quatro também se destacaram nas comunidades aqui estudadas (Leguminosae, Myrtaceae, Euphorbiaceae e Poaceae). Várias espécies pertencentes às 74 famílias referidas pelos citados autores, foram também registradas nas comunidades aqui estudadas, porém divergindo quanto ao número de citações. Os resultados, comparados com os obtidos em diversos trabalhos realizados no Brasil, com comunidades de diferentes perfis culturais, regiões e ecossistemas (Sales \& Lima 1985; Balée 1986; 1987; Kayner \& Duryea 1992; Begossi et al. 1993; Figueiredo et al. 1993; Albuquerque \& Chiappeta 1996/1997), demonstraram que as comunidades estudadas conhecem uma grande diversidade de vegetais, com diferentes formas de utilização (Tab. 1).

No entanto, muito desse conhecimento se refere às espécies cultivadas, correspondendo a 43,16\% na Usina São José (Ig), enquanto que a comunidade de Jaguarana, em Paulista, apresenta um índice mais elevado $(56,13 \%)$, devido ao seu perfil urbano, o que permite maior utilização de plantas cultivadas em jardins e quintais. Tais valores apontam para a existência de um processo de substituição dos recursos vegetais nativos por aqueles mais difundidos na sociedade em geral. De fato, as espécies mais citadas em cada comunidade são, na sua maioria, cultivadas, o que demonstra a importância que estas plantas vêm adquirindo em detrimento das nativas. Victor \& Andrade (1991) comentam que as plantas citadas com maior freqüência nos levantamentos que efetuaram em municípios da Zona Litoral-Mata de Pernambuco, tratavam-se de espécies não-típicas da vegetação ocorrente na região. Em outro estudo, Voeks (1996), realizando um levantamento da farmacopéia utilizada 
Tabela 1. Espécies utilizadas pelas comunidades da Usina São José (Ig - Igarassu) e Jaguarana (Pa - Paulista), nas seguintes categorias de uso: alimentação (a), comércio (b), construção (c), mágica (d), medicinal (e), tecnológica (f) e outros (g). H: hábito ${ }^{1}$; Fo: forma de obtenção ${ }^{2}$; Nc: número de coleta/Alberto Silva. ${ }^{1} \mathrm{Ab}$ : arbustivo, Av: arbóreo, E: herbáceo, L: liana; ${ }^{2} \mathrm{C}$ : cultivada, I: introduzida, N: nativa, O: comprada; *Dicotiledônea, **Monocotiledônea ***Pteridófita

\begin{tabular}{|c|c|c|c|c|c|}
\hline Táxon & $\mathrm{H}$ & Fo & Ig & $\mathrm{Pa}$ & $\mathrm{Nc}$ \\
\hline \multicolumn{6}{|l|}{ ACANTHACEAE* } \\
\hline Justicia pectoralis Jacq. (chambá) & $\mathrm{E}$ & $\mathrm{C}$ & $\mathrm{e}$ & $\mathrm{e}$ & 139,238 \\
\hline Justicia sp. (anador) & $\mathrm{E}$ & $\mathrm{C}$ & $\mathrm{e}$ & $\mathrm{e}$ & 215,250 \\
\hline \multicolumn{6}{|l|}{ AGAVACEAE** } \\
\hline Sanseviera sp. (espada de são jorge) & $\mathrm{E}$ & $\mathrm{C}$ & $\mathrm{g}$ & g & \\
\hline \multicolumn{6}{|l|}{ AMARANTHACEAE* } \\
\hline Pfaffia glomerata (Spreng.) Pedersen (acônito) & $\mathrm{E}$ & $\mathrm{C}$ & $\mathrm{e}$ & de & 152,232 \\
\hline Sem determinação (bredo) & $\mathrm{E}$ & $\mathrm{C}$ & $\mathrm{a}$ & - & \\
\hline \multicolumn{6}{|l|}{ AMARYLLIDACEAE** } \\
\hline Amaryllis belladona L. (cebola-xenxém) & $\mathrm{E}$ & $\mathrm{C}$ & $\mathrm{e}$ & - & 96 \\
\hline \multicolumn{6}{|l|}{ ANACARDIACEAE* } \\
\hline Anacardium occidentale L. (caju, caju-roxo) & Av & $\mathrm{N}$ & aeg & abe & \\
\hline Mangifera indica L. (manga) & Av & $\mathrm{C}$ & aef & abdf & 120 \\
\hline Schinus terebintifolius Raddi (aroeira) & $\mathrm{Av}$ & $\mathrm{N}$ & ce & de & 223 \\
\hline Spondias mombim L. (cajá) & $\mathrm{Av}$ & $\mathrm{N}$ & a & af & 168 \\
\hline S. tuberosa Arruda (imbu) & $\mathrm{Av}$ & $\mathrm{C}$ & $\mathrm{a}$ & - & 80 \\
\hline Spondias sp. (cajarana, ciriguela) & Av & $\mathrm{C}$ & $\mathrm{a}$ & $\mathrm{a}$ & 111,248 \\
\hline Tapirira guianensis Aubl. (cupiúba, pau-pombo) & $\mathrm{Av}$ & $\mathrm{N}$ & $\mathrm{cf}$ & $\mathrm{cf}$ & 125,166 \\
\hline \multicolumn{6}{|l|}{ ANNONACEAE* } \\
\hline Annona marcgravii Mart. (aticum, aticum-cagão) & Av & $\mathrm{C}$ & aeg & ae & 24,164 \\
\hline A. muricata L. (graviola) & Av & $\mathrm{C}$ & $\mathrm{a}$ & ae & \\
\hline A. squamosa L. (pinha) & Av & $\mathrm{C}$ & $\mathrm{a}$ & $\mathrm{a}$ & 140 \\
\hline Guatteria australis A.St.-Hil. (embira-preta) & Av & $\mathrm{N}$ & $\mathrm{cf}$ & $\mathrm{c}$ & 82 \\
\hline Xylopia frutescens Aubl. (embira-vermelha) & Av & $\mathrm{N}$ & $\mathrm{f}$ & $\mathrm{f}$ & 77 \\
\hline Sem determinação (aticum-da-mata) & $\mathrm{Av}$ & $\mathrm{N}$ & ae & - & \\
\hline Sem determinação (mium) & Av & $\mathrm{N}$ & $\mathrm{c}$ & - & \\
\hline \multicolumn{6}{|l|}{ APIACEAE* } \\
\hline Coriandrum sativum L. (coentro) & $\mathrm{E}$ & $\mathrm{C}$ & abe & $\mathrm{ab}$ & \\
\hline Daucus carota L. (cenoura) & $\mathrm{E}$ & $\mathrm{C}$ & ae & $\mathrm{a}$ & \\
\hline Pimpinella anisum L. (erva-doce) & $\mathrm{E}$ & $\mathrm{C}$ & de & $\mathrm{e}$ & \\
\hline Sem determinação (pé-de-dinheiro) & $\mathrm{E}$ & $\mathrm{C}$ & $\mathrm{g}$ & - & \\
\hline \multicolumn{6}{|l|}{ APOCYNACEAE* } \\
\hline Aspidosperma sp. (pitiá) & $A v$ & $\mathrm{~N}$ & $\mathrm{f}$ & - & \\
\hline Catharanthus roseos G. Don. (boa-noite) & $\mathrm{E}$ & $\mathrm{C}$ & e & g & 217 \\
\hline Ervatamia coronaria (Jacq.) Stapf (jasmim) & $\mathrm{Ab}$ & $\mathrm{C}$ & $\mathrm{g}$ & $\mathrm{g}$ & 224 \\
\hline Ervatamia sp. 1 (jasmim-cambraia) & $\mathrm{Ab}$ & $\mathrm{C}$ & - & $\begin{array}{l}\mathrm{g} \\
\mathrm{g}\end{array}$ & \\
\hline Ervatamia sp. 2 (jasmim-pera) & $\mathrm{Ab}$ & $\mathrm{C}$ & - & $\mathrm{g}$ & \\
\hline Hancornia speciosa Gomez (mangaba) & Av & $\mathrm{N}$ & a & ace & 180 \\
\hline Himatanthus phagedaenicus (Mart.) Woodson (banana-de-papagaio) & Av & $\mathrm{N}$ & $\mathrm{f}$ & - & 177,198 \\
\hline Plumeria sp. (jasmim-vapor) & $\mathrm{Ab}$ & $\mathrm{C}$ & - & $\mathrm{g}$ & \\
\hline \multicolumn{6}{|l|}{ ARACEAE** } \\
\hline Caladium bicolor (Aiton) Vent. (tinhorão) & $\mathrm{E}$ & $\mathrm{N}$ & $\mathrm{e}$ & e & 258 \\
\hline Dieffenbachia picta (Lodd.) Schott (comigo-ninguém-pode) & $\mathrm{E}$ & $\mathrm{C}$ & $\mathrm{g}$ & $\mathrm{g}$ & \\
\hline Philodendron sp. 1 (imbé) & $\mathrm{E}$ & $\mathrm{N}$ & eg & eg & 260 \\
\hline Philodendron sp. 2 (imbé-de-fonte) & $\mathrm{E}$ & $\mathrm{N}$ & eg & $\mathrm{g}$ & 261 \\
\hline Scindapus aureus Engl. (jibóia) & $\mathrm{E}$ & $\mathrm{C}$ & - & g & \\
\hline Sem determinação (pena-de-urubu) & $\mathrm{E}$ & $\mathrm{C}$ & g & - & \\
\hline \multicolumn{6}{|l|}{ ARALIACEAE* } \\
\hline Schefflera sp. (chefrera) & $\mathrm{E}$ & $\mathrm{C}$ & - & $\mathrm{g}$ & \\
\hline \multicolumn{6}{|l|}{ ARECACEAE ** } \\
\hline Acrocomia intumescens Drude (macaíba) & Av & $\mathrm{N}$ & abe & $\mathrm{a}$ & \\
\hline Bactris sp. 1 (coco-de-fuso) & $\mathrm{Av}$ & $\mathrm{N}$ & $\mathrm{ab}$ & $\mathrm{a}$ & \\
\hline Bactris sp. 2 (maraial) & $\mathrm{E}$ & $\mathrm{N}$ & af & - & \\
\hline Cocos nucifera L. (coco) & $\mathrm{Av}$ & $\mathrm{C}$ & abe & ace & \\
\hline
\end{tabular}


Tabela 1 (continuação)

\begin{tabular}{|c|c|c|c|c|c|}
\hline Táxon & $\mathrm{H}$ & Fo & Ig & $\mathrm{Pa}$ & $\mathrm{Nc}$ \\
\hline \multicolumn{6}{|l|}{ ARECACEAE $* *$} \\
\hline Desmoncus sp. (titara) & $\mathrm{E}$ & $\mathrm{N}$ & $\mathrm{a}$ & - & \\
\hline Elaeis guineensis L. (dendê) & Av & $\mathrm{I}$ & a & af & \\
\hline Syagrus sp. (catolé, coco-babão) & Av & $\mathrm{N}$ & a & - & \\
\hline Sem determinação (palmeira) & $\mathrm{E}$ & $\mathrm{C}$ & $\mathrm{dg}$ & $\mathrm{g}$ & \\
\hline \multicolumn{6}{|l|}{ ASTERACEAE* } \\
\hline Acanthospermum hispidum DC. (espinho de cigano) & $\mathrm{E}$ & $\mathrm{N}$ & e & e & 63 \\
\hline Artemisia sp. (artemísia, anador) & $\mathrm{E}$ & $\mathrm{C}$ & $\mathrm{e}$ & - & \\
\hline Coniza bonariensis (L.) Cronquist (rabo-de-raposa) & $\mathrm{E}$ & $\mathrm{N}$ & e & e & \\
\hline Helianthus annuus L. (girassol) & $\mathrm{E}$ & $\mathrm{C}$ & e & e & \\
\hline Lactuca sativa L. (alface) & $\mathrm{E}$ & $\mathrm{C}$ & $\mathrm{ab}$ & a & \\
\hline Vernonia condensata Toledo (alcachofra) & $\mathrm{Ab}$ & $\mathrm{C}$ & $\mathrm{e}$ & e & 26 \\
\hline Zinnia elegans Jacq. (benedita) & $\mathrm{E}$ & $\mathrm{C}$ & - & $\mathrm{g}$ & 216 \\
\hline \multicolumn{6}{|l|}{ BEGONIACEAE* } \\
\hline Begonia vitifolia Schott (capeba) & $\mathrm{E}$ & $\mathrm{C}$ & e & - & \\
\hline \multicolumn{6}{|l|}{ BIGNONIACEAE* } \\
\hline Tabebuia avellanedae Lorenz et Gris. (pau-d'arco-roxo) & Av & $\mathrm{N}$ & cef & ce & \\
\hline \multicolumn{6}{|l|}{ BIXACEAE* } \\
\hline Bixa orellana L. (urucum) & Av & $\mathrm{C}$ & ae & - & \\
\hline \multicolumn{6}{|l|}{ BLECNACEAE*** } \\
\hline Blecnum serrulatum Rich. (avenca) & $\mathrm{E}$ & $\mathrm{N}$ & $\mathrm{g}$ & - & 89 \\
\hline \multicolumn{6}{|l|}{ BOMBACACEAE* } \\
\hline Pachira aquatica Aubl. (castanhola) & Av & $\mathrm{C}$ & $\mathrm{ac}$ & - & 98 \\
\hline Sem determinação (munguba) & Av & $\mathrm{N}$ & $\mathrm{f}$ & - & \\
\hline \multicolumn{6}{|l|}{ BORAGINACEAE* } \\
\hline Cordia tokeve Aubl. (gagaúba) & Av & $\mathrm{N}$ & $\mathrm{f}$ & - & 208 \\
\hline Heliotropium indicum L. (fedegoso) & $\mathrm{E}$ & $\mathrm{I}$ & e & eg & 39,137 \\
\hline \multicolumn{6}{|l|}{ BRASSICACEAE* } \\
\hline Brassica oleracea L. (couve) & $\mathrm{E}$ & $\mathrm{C}$ & ae & ae & \\
\hline B. oleracea L. (repolho) & $\mathrm{E}$ & $\mathrm{C}$ & $\mathrm{ab}$ & - & \\
\hline Nasturtium officinale R. Br. (agrião) & $\mathrm{E}$ & $\mathrm{C}$ & ae & e & 209 \\
\hline \multicolumn{6}{|l|}{ BROMELIACEAE** } \\
\hline Aechmea sp. (gravatá) & $\mathrm{E}$ & $\mathrm{N}$ & a & - & 211 \\
\hline Ananas sativus Schult. (abacaxi) & $\mathrm{E}$ & $\mathrm{C}$ & a & a & \\
\hline \multicolumn{6}{|l|}{ BURSERACEAE* } \\
\hline Protium heptaphyllum (Aubl.) Marchi (amescla) & Av & $\mathrm{N}$ & e & $\mathrm{c}$ & 106,119 \\
\hline \multicolumn{6}{|l|}{ CACTACEAE* } \\
\hline Cereus jamacaru DC. (mandacaru) & $\mathrm{E}$ & $\mathrm{C}$ & e & e & \\
\hline \multicolumn{6}{|l|}{ CAMPANULACEAE* } \\
\hline Centropogon cornutus (L.) Druce (sorriso) & $\mathrm{E}$ & $\mathrm{C}$ & $\mathrm{g}$ & $\mathrm{g}$ & 123 \\
\hline \multicolumn{6}{|l|}{ CAPPARACEAE* } \\
\hline Cleome spinosa Jacq. (muçambê) & $\mathrm{E}$ & $\mathrm{N}$ & e & e & 37,178 \\
\hline \multicolumn{6}{|l|}{ CAPRIFOLIACEAE* } \\
\hline Sambucus australis Cham. \& Schltr. (sabugo) & $\mathrm{Ab}$ & $\mathrm{C}$ & e & e & \\
\hline \multicolumn{6}{|l|}{ CARICACEAE* } \\
\hline Carica papaya L. (mamão, mamão-de-corda, mamão-caiana) & Av & $\mathrm{C}$ & ae & abe & 70 \\
\hline \multicolumn{6}{|l|}{ CECROPIACEAE* } \\
\hline Cecropia palmata Willd. (imbaúba-branca) & Av & $\mathrm{N}$ & $\mathrm{f}$ & af & 71 \\
\hline CELASTRACEAE* & & & & & \\
\hline Maytenus rigida Mart. (dourado, bonome) & Av & I & e & - & \\
\hline CHENOPODIACEAE* & & & & & \\
\hline Beta vulgaris L. (beterraba) & $\mathrm{E}$ & $\mathrm{C}$ & ae & a & \\
\hline Chenopodium ambrosioides L. (mastruz) & $\mathrm{E}$ & $\mathrm{C}$ & $\mathrm{e}$ & $\mathrm{e}$ & \\
\hline CHRYSOBALANACEAE* & & & & & \\
\hline Chrysobalanus icaco L. (guajiru) & $\mathrm{Ab}$ & $\mathrm{N}$ & - & ae & 60,110 \\
\hline Sem determinação (oiti, oiti-coró) & Av & $\mathrm{N}$ & abcf & $\mathrm{a}$ & \\
\hline Sem determinação (oiti-branco) & Av & $\mathrm{N}$ & $\mathrm{a}$ & - & \\
\hline
\end{tabular}


Tabela 1 (continuação)

\begin{tabular}{|c|c|c|c|c|c|}
\hline Táxon & $\mathrm{H}$ & Fo & $\mathrm{Ig}$ & $\mathrm{Pa}$ & $\mathrm{Nc}$ \\
\hline \multicolumn{6}{|l|}{ CHRYSOBALANACEAE* } \\
\hline Sem determinação (oiti-liso) & $\mathrm{Av}$ & $\mathrm{N}$ & $\mathrm{a}$ & - & \\
\hline Sem determinação (oiti-pequeno) & Av & $\mathrm{N}$ & $\mathrm{a}$ & - & \\
\hline \multicolumn{6}{|l|}{ CLUSIACEAE* } \\
\hline Symphonia sp. (bulandi) & Av & $\mathrm{N}$ & $\mathrm{f}$ & $\mathrm{cf}$ & \\
\hline Vismia guianensis (Aubl.) Choisy (lacre) & $\mathrm{Ab}$ & $\mathrm{N}$ & $\mathrm{e}$ & cef & 88,108 \\
\hline Sem determinação (camaçari) & Av & $\mathrm{N}$ & $\mathrm{f}$ & $\mathrm{f}$ & \\
\hline \multicolumn{6}{|l|}{ COMBRETACEAE* } \\
\hline Buchenavia sp. (embiridiba) & Av & $\mathrm{N}$ & $\mathrm{c}$ & - & 252 \\
\hline Terminalia catappa L. (coração-de-nego) & Av & $\mathrm{C}$ & ac & $\mathrm{ad}$ & 121 \\
\hline \multicolumn{6}{|l|}{ CONVOLVULACEAE* } \\
\hline Ipomoea asarifolia (Ders.) R. \& Sch. (salsa) & $\mathrm{L}$ & I & - & de & \\
\hline Ipomoea batatas Poir. (batata-doce) & $\mathrm{E}$ & $\mathrm{C}$ & abe & $a b$ & \\
\hline Operculina alata (Ham.) Urb. (batata-de-purga) & $\mathrm{L}$ & $\mathrm{N}$ & $\mathrm{e}$ & - & 173 \\
\hline Sem determinação (acanfor, acançu) & $\mathrm{E}$ & $\mathrm{N}$ & de & e & 221 \\
\hline \multicolumn{6}{|l|}{ CRASSULACEAE* } \\
\hline Kalanchoe brasiliensis Cambess. (corona) & $\mathrm{E}$ & $\mathrm{C}$ & $\mathrm{e}$ & $\mathrm{e}$ & \\
\hline \multicolumn{6}{|l|}{ CUCURBITACEAE* } \\
\hline Citrullus vulgaris Schrad. (melancia) & $\mathrm{L}$ & $\mathrm{C}$ & $a b$ & ae & \\
\hline Cucumis anguria L. (maxixe) & $\mathrm{E}$ & $\mathrm{C}$ & $a b$ & $\mathrm{a}$ & \\
\hline C. melo L. (melão) & $\mathrm{L}$ & $\mathrm{C}$ & - & a & \\
\hline C. sativus L. (pepino) & $\mathrm{E}$ & $\mathrm{O}$ & $\mathrm{a}$ & - & \\
\hline Curcubita pepo L. (jerimum) & $\mathrm{L}$ & $\mathrm{C}$ & $a b$ & a & \\
\hline Luffa operculata Cong. (cabacinho) & $\mathrm{L}$ & $\mathrm{O}$ & $\mathrm{e}$ & - & \\
\hline Momordica charantia L. (melão-de-são-caetano, melão-do-mato) & $\mathrm{L}$ & $\mathrm{N}$ & ae & $\mathrm{e}$ & 41,100 \\
\hline Sechium edule Sw. (chuchu) & $\mathrm{L}$ & $\mathrm{C}$ & ae & $\mathrm{a}$ & \\
\hline Sem determinação (maxixe-do-mato) & $\mathrm{E}$ & $\mathrm{N}$ & - & - & \\
\hline \multicolumn{6}{|l|}{ CYPERACEAE** } \\
\hline Cyperum sp. (munimbu) & $\mathrm{E}$ & $\mathrm{N}$ & $\mathrm{c}$ & - & 245 \\
\hline Eleocharis interstincta (Vahl) R. \& S. (junco) & $\mathrm{E}$ & I & $\mathrm{g}$ & - & 73 \\
\hline Rhinchospora ciliata (Vahl) Kük. (capim-estrela) & $\mathrm{E}$ & $\mathrm{N}$ & $\mathrm{e}$ & - & 124 \\
\hline \multicolumn{6}{|l|}{ DAVALLIACEAE*** } \\
\hline Davallia sp. (canela-de-veado) & $\mathrm{E}$ & $\mathrm{C}$ & $\mathrm{g}$ & $\mathrm{g}$ & 240 \\
\hline Nephrolepis sp. (avenca) & $\mathrm{E}$ & $\mathrm{C}$ & $\mathrm{g}$ & $\mathrm{g}$ & \\
\hline \multicolumn{6}{|l|}{ DENNSTAEDTIACEAE*** } \\
\hline Pteridium aquilinum (L.) Kuhn (avenca) & $\mathrm{E}$ & $\mathrm{N}$ & $\mathrm{g}$ & - & 97 \\
\hline \multicolumn{6}{|l|}{ DILLENIACEAE* } \\
\hline Davilla kunthii A.St.-Hil. (cipó-de-fogo) & $\mathrm{L}$ & $\mathrm{N}$ & - & $\mathrm{f}$ & 135 \\
\hline \multicolumn{6}{|l|}{ DIOSCOREACEAE** } \\
\hline Dioscorea sp. 1 (inhame) & $\mathrm{E}$ & $\mathrm{C}$ & $a b$ & $\mathrm{ab}$ & \\
\hline Dioscorea sp. 2 (cará) & $\mathrm{E}$ & $\mathrm{C}$ & $\mathrm{a}$ & - & \\
\hline \multicolumn{6}{|l|}{ ELAEOCARPACEAE* } \\
\hline Sem determinação (mamajuda) & Av & $\mathrm{N}$ & $\mathrm{cf}$ & - & \\
\hline \multicolumn{6}{|l|}{ EUPHORBIACEAE* } \\
\hline Cnidosculus urens (L.) Arthur (urtiga-branca) & $\mathrm{E}$ & $\mathrm{N}$ & $\mathrm{e}$ & $\mathrm{e}$ & 113,188 \\
\hline Croton sp. 1 (crote) & $\mathrm{Ab}$ & $\mathrm{C}$ & $\mathrm{fg}$ & $\mathrm{fg}$ & 154 \\
\hline Croton sp. 2 (crote-molambo) & $\mathrm{Ab}$ & $\mathrm{C}$ & - & fg & 155 \\
\hline Croton sp. 3 (marmeleiro) & - & $\mathrm{O}$ & - & $\mathrm{e}$ & \\
\hline Euphorbia thymifolia L. (quebra-pedra, pé-de-pombo) & $\mathrm{E}$ & $\mathrm{N}$ & $\mathrm{e}$ & - & \\
\hline Jatropha gossypiifolia L. (pinhão-roxo) & $\mathrm{Ab}$ & $\mathrm{C}$ & de & $\mathrm{d}$ & 56,148 \\
\hline J. molissima (Pohl) Baill. (pinhão-branco) & $\mathrm{Ab}$ & $\mathrm{C}$ & de & de & 150,244 \\
\hline Manihot esculenta Crantz (macaxeira) & $\mathrm{E}$ & $\mathrm{C}$ & $a b$ & abe & 40 \\
\hline M. esculenta Crantz (mandioca) & $\mathrm{E}$ & $\mathrm{C}$ & $a b$ & $\mathrm{ab}$ & \\
\hline Pera ferruginea Müll. Arg. (chumbinho-madeira) & Av & $\mathrm{N}$ & $\mathrm{f}$ & - & 86 \\
\hline Phyllanthus amarus Schum. \& Thonn. (quebra-pedra) & $\mathrm{E}$ & $\mathrm{N}$ & $\mathrm{e}$ & $\mathrm{e}$ & 229 \\
\hline Pogonophora sp. (cocão) & $\mathrm{Av}$ & $\mathrm{N}$ & $\mathrm{cf}$ & $\mathrm{cf}$ & $\begin{array}{l}112 \\
\text { contiv }\end{array}$ \\
\hline
\end{tabular}


Tabela 1 (continuação)

\begin{tabular}{|c|c|c|c|c|c|}
\hline Táxon & $\mathrm{H}$ & Fo & Ig & $\mathrm{Pa}$ & $\mathrm{Nc}$ \\
\hline \multicolumn{6}{|l|}{ EUPHORBIACEAE* } \\
\hline Ricinus communis L. (carrapateira) & $\mathrm{Ab}$ & I & $\mathrm{d}$ & $\mathrm{e}$ & 91 \\
\hline \multicolumn{6}{|l|}{ FLACOURTIACEAE* } \\
\hline Casearia sylvestris Eicher. (caiubim-branca) & $\mathrm{Ab}$ & $\mathrm{N}$ & $\mathrm{e}$ & - & 255 \\
\hline \multicolumn{6}{|l|}{ HELICONIACEAE** } \\
\hline Heliconia psittacorum L. (paquivira) & $\mathrm{E}$ & $\mathrm{N}$ & $\mathrm{g}$ & g & 74,184 \\
\hline \multicolumn{6}{|l|}{ HERNANDIACEAE* } \\
\hline Sparanttanthelium botucudorum Mart. (camundongo) & $\mathrm{Av}$ & $\mathrm{N}$ & $\mathrm{c}$ & - & 76 \\
\hline \multicolumn{6}{|l|}{ ILLICIACEAE* } \\
\hline Illicium verum Hook. (aniz-estrelado) & $\mathrm{E}$ & $\mathrm{C}$ & $\mathrm{e}$ & - & \\
\hline \multicolumn{6}{|l|}{ LAMIACEAE* } \\
\hline Aeolanthus suaveolens Mart. ex Spreng. (macassá) & $\mathrm{E}$ & $\mathrm{C}$ & de & $\mathrm{d}$ & 231 \\
\hline Mentha pulegium L. (vaporub, vique) & $\mathrm{E}$ & $\mathrm{C}$ & - & $\mathrm{e}$ & 160 \\
\hline M. $x$ villosa Huds. (hortelã-miúda) & $\mathrm{E}$ & $\mathrm{C}$ & ae & ae & 227 \\
\hline Ocimum americanum L. (manjerona) & $\mathrm{E}$ & I & $\mathrm{d}$ & - & \\
\hline O. basilicum L. var. minimum (manjericão-miudinho) & $\mathrm{E}$ & $\mathrm{C}$ & $\mathrm{e}$ & e & 141,226 \\
\hline Ocimum campechianum L. (alfavaca-de-cheiro) & $\mathrm{E}$ & $\mathrm{I}$ & - & $\mathrm{d}$ & 219 \\
\hline O. gratissimum L. (alfavaca) & $\mathrm{E}$ & I & de & de & 25,54 \\
\hline Ocimum sp. (manjericão) & $\mathrm{E}$ & $\mathrm{C}$ & ade & de & \\
\hline Plectranthus amboinicus Lour. (hortelã-graúda, hortelã-da-folha grossa) & $\mathrm{E}$ & $\mathrm{C}$ & $\mathrm{e}$ & e & 233 \\
\hline P. barbatus Andr. (boldo) & $\mathrm{E}$ & $\mathrm{D}$ & $\mathrm{e}$ & $\mathrm{e}$ & 90 \\
\hline Rosmarinus officinalis L. (alecrim) & $\mathrm{E}$ & $\mathrm{C}$ & de & de & \\
\hline Sem determinação (hortelã-caboclo, hortelã-fernandes, malva-santa) & $\mathrm{E}$ & $\mathrm{C}$ & $\mathrm{e}$ & e & 156,230 \\
\hline Sem determinação (hortelã-pastilha) & $\mathrm{E}$ & $\mathrm{C}$ & $\mathrm{e}$ & - & \\
\hline Sem determinação (hortelã-pimenta) & $\mathrm{E}$ & $\mathrm{C}$ & $\mathrm{e}$ & - & \\
\hline Sem determinação (verga-morta) & $\mathrm{E}$ & $\mathrm{C}$ & $\mathrm{e}$ & $\mathrm{e}$ & 161 \\
\hline \multicolumn{6}{|l|}{ LAURACEAE* } \\
\hline Nectandra cuspidata (Ness \& Mart.) Ness (canela) & Av & $\mathrm{N}$ & $\mathrm{e}$ & $\mathrm{e}$ & 138 \\
\hline Ocotea glomerata (Ness) Mez (louro) & $\mathrm{Av}$ & $\mathrm{N}$ & ae & $\mathrm{c}$ & 75 \\
\hline Persea americana Mill. (abacate) & Av & $\mathrm{C}$ & ae & $\mathrm{a}$ & 32 \\
\hline Sem determinação (camundongo) & Av & $\mathrm{N}$ & - & $\mathrm{f}$ & 257 \\
\hline \multicolumn{6}{|l|}{ LECYTHIDACEAE* } \\
\hline Eschweilera ovatta (Cambess.) Miers (embiriba) & Av & $\mathrm{N}$ & $\mathrm{cf}$ & cef & 84,175 \\
\hline Gustavia augusta L. (japaranduba) & $\mathrm{Av}$ & $\mathrm{N}$ & $\mathrm{f}$ & - & 34,197 \\
\hline Lecythis sp. (sapucaia) & $\mathrm{Av}$ & $\mathrm{N}$ & $\mathrm{cf}$ & $\mathrm{a}$ & \\
\hline \multicolumn{6}{|l|}{ LEG. CAESALPINIOIDEAE* } \\
\hline Apuleia sp. (jitaí) & $\mathrm{Av}$ & $\mathrm{N}$ & $\mathrm{cf}$ & $\mathrm{a}$ & 253 \\
\hline Bauhinia monandra Kurz (pata-de-vaca) & Av & $\mathrm{C}$ & - & $\mathrm{e}$ & \\
\hline Caesalpinia echinata Lam. (pau-brasil) & $\mathrm{Av}$ & $\mathrm{N}$ & - & $\mathrm{g}$ & 167 \\
\hline C. ferrea Mart. ex Tul. (jucá, pau-ferro) & $\mathrm{Av}$ & $\mathrm{I}$ & - & - & \\
\hline C. pyramidalis Tul. (catingueira) & Av & $\mathrm{O}$ & $\mathrm{e}$ & $\mathrm{e}$ & \\
\hline Copaifera sp. (pau-d'óleo) & Av & $\mathrm{N}$ & $\mathrm{e}$ & - & \\
\hline Hymenaea martiana Hayne (jatobá) & $\mathrm{Av}$ & $\mathrm{N}$ & $\mathrm{ac}$ & ae & 181 \\
\hline Senna alata $($ L.) Link (café-beirão) & $\mathrm{Ab}$ & $\mathrm{N}$ & $\mathrm{e}$ & - & \\
\hline S. georgica Irwin \& Barneby (lava-prato) & $\mathrm{Ab}$ & $\mathrm{N}$ & $\mathrm{e}$ & $\mathrm{d}$ & 44,128 \\
\hline S. occidentalis (L.) Link (manjerioba) & $\mathrm{E}$ & $\mathrm{N}$ & $\mathrm{e}$ & de & 53 \\
\hline Tamarindus indica L. (tamarindo) & Av & $\mathrm{C}$ & $\mathrm{e}$ & - & 176 \\
\hline Sem determinação (coração-de-nego) & $\mathrm{Av}$ & $\mathrm{N}$ & $\mathrm{cf}$ & cef & \\
\hline \multicolumn{6}{|l|}{ LEG. MIMOSOIDEAE* } \\
\hline Anadenanthera macrocarpa (Benth.) Brenan (angico) & $\mathrm{Av}$ & $\mathrm{O}$ & $\mathrm{e}$ & - & \\
\hline Inga bahienses Benth. (ingá-porco) & $\mathrm{Av}$ & $\mathrm{N}$ & acf & af & 207 \\
\hline I. fagifolia (L.) Willd. (ingaí) & Av & $\mathrm{N}$ & $\mathrm{a}$ & - & 92,172 \\
\hline I. thibaudiana DC. (ingá) & $\mathrm{Av}$ & $\mathrm{N}$ & acf & - & 85 \\
\hline Inga sp. (ingá-caixão) & $\mathrm{Av}$ & $\mathrm{N}$ & $\mathrm{a}$ & - & 129,241 \\
\hline Mimosa invisa Mart. (malícia) & $\mathrm{E}$ & $\mathrm{N}$ & $\mathrm{e}$ & $\mathrm{e}$ & \\
\hline Mimosa sp. (jurema-preta, jurema-do-sertão) & Av & $\mathrm{C}$ & - & $\mathrm{f}$ & 162 \\
\hline
\end{tabular}


Tabela 1 (continuação)

\begin{tabular}{|c|c|c|c|c|c|}
\hline Táxon & $\mathrm{H}$ & Fo & $\mathrm{Ig}$ & $\mathrm{Pa}$ & $\mathrm{Nc}$ \\
\hline \multicolumn{6}{|l|}{ LEG. MIMOSOIDEAE* } \\
\hline Parkia pendula Benth. (visgueiro) & Av & $\mathrm{N}$ & $\mathrm{cf}$ & - & \\
\hline Pithecellobium cochliocarpum (Gomez) Macbr. (barbatenon) & Av & $\mathrm{N}$ & ce & ce & 47 \\
\hline P. dumosum Benth. (jurema) & Av & $\mathrm{N}$ & $\mathrm{c}$ & - & 72 \\
\hline P. pedicellare (DC.) Benth. (jaguarana) & Av & $\mathrm{N}$ & $\mathrm{cf}$ & $\mathrm{g}$ & \\
\hline P. saman (Jacq.) Baill. var. acuntifolium Benth. (bordão-de-velho) & Av & $\mathrm{N}$ & $\mathrm{c}$ & - & 64 \\
\hline Schrankia leptocarpa DC. (malícia) & $\mathrm{E}$ & $\mathrm{N}$ & $\mathrm{e}$ & $\mathrm{e}$ & 35,213 \\
\hline Stryphnodendron sp. (favinha) & Av & $\mathrm{N}$ & $\mathrm{f}$ & - & \\
\hline Sem determinação (camundongo) & Av & $\mathrm{N}$ & - & $\mathrm{g}$ & \\
\hline \multicolumn{6}{|l|}{ LEG. PAPILIONOIDEAE* } \\
\hline Andira fraxinifolia Benth. (angelim-roxo) & Av & $\mathrm{N}$ & - & ef & \\
\hline A. nitida Mart. (angelim, angelim-amargoso, angelim-de-pedra) & Av & $\mathrm{N}$ & $\mathrm{cf}$ & - & 62,247 \\
\hline Andira sp. (angelim-doce) & Av & $\mathrm{N}$ & $\mathrm{c}$ & - & \\
\hline Arachis hipogaea L. (amendoim ) & $\mathrm{E}$ & $\mathrm{C}$ & $a b$ & $\mathrm{a}$ & \\
\hline Bowdichia virgilioides Humb., Bonplan \& Kunth. (sucupira) & Av & $\mathrm{N}$ & cef & cef & 46,130 \\
\hline Cajanus cajan (L.) Mill. (feijão-guandu) & $\mathrm{E}$ & $\mathrm{C}$ & - & abe & \\
\hline Clitoria fairchildiana Howard (sombreiro) & Av & $\mathrm{C}$ & $\mathrm{g}$ & $\mathrm{g}$ & 2246 \\
\hline Erytrina vellutina Willd. (mulungu) & Av & $\mathrm{C}$ & $\mathrm{e}$ & $\mathrm{e}$ & 183 \\
\hline Lonchocarpus sericeus Humb., Bonplan \& Kunth (piaca) & Av & $\mathrm{N}$ & $\mathrm{cf}$ & - & 116 \\
\hline Machaerium hirtum (Vell.) Stellfeld (espinho-de-judeu) & Av & $\mathrm{N}$ & $\mathrm{e}$ & - & 243 \\
\hline Phaseolus vulgaris L. (feijão-de-arranque) & $\mathrm{L}$ & $\mathrm{C}$ & $\mathrm{a}$ & - & \\
\hline Vigna sinensis Endl. (feijão-de-corda) & $\mathrm{L}$ & $\mathrm{C}$ & $a b$ & $\mathrm{a}$ & \\
\hline Sem determinação (sucupira-mirim) & Av & $\mathrm{N}$ & $\mathrm{cf}$ & - & \\
\hline Sem determinação (sucupira-preta) & Av & $\mathrm{N}$ & ef & - & \\
\hline \multicolumn{6}{|l|}{ LILIACEAE** } \\
\hline Allium ascalonicum L. (cebolinha-branca) & $\mathrm{E}$ & $\mathrm{C}$ & ae & - & \\
\hline A. cepa L. (cebola) & $\mathrm{E}$ & $\mathrm{C}$ & ae & $\mathrm{a}$ & \\
\hline A. schoenoprasum L. (cebolinha) & $\mathrm{E}$ & $\mathrm{C}$ & abe & $\mathrm{a}$ & \\
\hline Aloe vera L. (babosa) & $\mathrm{E}$ & $\mathrm{C}$ & $\mathrm{e}$ & $\mathrm{e}$ & \\
\hline Lilium sp. (lírio) & $\mathrm{E}$ & $\mathrm{C}$ & - & $\mathrm{dg}$ & \\
\hline \multicolumn{6}{|l|}{ LORANTHACEAE* } \\
\hline $\begin{array}{l}\text { Phthirusa pyrofolia (Humb., Bonplan \& Kunth Eichler } \\
\quad \text { (esterco-de-passarinho) }\end{array}$ & $\mathrm{L}$ & $\mathrm{N}$ & $\mathrm{e}$ & - & 122 \\
\hline \multicolumn{6}{|l|}{ LYCOPODIACEAE*** } \\
\hline Lycopodiella cernua (L.) Pic. Serm. (unha-de-gato) & $\mathrm{E}$ & $\mathrm{N}$ & $\mathrm{g}$ & - & \\
\hline \multicolumn{6}{|l|}{ MALPIGHIACEAE* } \\
\hline Byrsonima sericea DC. (murici) & Av & $\mathrm{N}$ & $\mathrm{cf}$ & $\mathrm{cf}$ & 118,134 \\
\hline Malpighia emarginata DC. (acerola) & Av & $\mathrm{C}$ & ae & $\mathrm{a}$ & 55 \\
\hline \multicolumn{6}{|l|}{ MALVACEAE* } \\
\hline Gossypium barbadense L. (algodão) & $\mathrm{Ab}$ & $\mathrm{C}$ & be & - & 43 \\
\hline Gossypium sp. (algodão-do-mato) & Av & $\mathrm{N}$ & $\mathrm{e}$ & - & 67 \\
\hline Hibiscus esculentus L. (quiabo) & $\mathrm{E}$ & $\mathrm{C}$ & $a b$ & $\mathrm{a}$ & \\
\hline H. rosa-sinensis L. (papoula) & $\mathrm{Ab}$ & $\mathrm{C}$ & fg & $\mathrm{fg}$ & 147 \\
\hline Malva sp. (malva-rosa) & $\mathrm{E}$ & $\mathrm{N}$ & $\mathrm{e}$ & - & \\
\hline Sida rhombifolia L. (relógio) & $\mathrm{E}$ & $\mathrm{N}$ & - & - & 57 \\
\hline \multicolumn{6}{|l|}{ MARANTACEAE** } \\
\hline Ischnosiphon sp. (uruba) & $\mathrm{E}$ & $\mathrm{N}$ & $\mathrm{F}$ & - & \\
\hline \multicolumn{6}{|l|}{ MELASTOMATACEAE* } \\
\hline Clidemia spicata DC. (remela-de-velho) & $\mathrm{Ab}$ & $\mathrm{N}$ & $\mathrm{f}$ & ae & 131 \\
\hline Miconia prasina (Sw.) DC. (sabiazeira) & Av & $\mathrm{N}$ & $\mathrm{fg}$ & - & 36 \\
\hline Miconia sp. (carrasco) & $\mathrm{Ab}$ & $\mathrm{N}$ & $\mathrm{e}$ & $\mathrm{d}$ & 114,190 \\
\hline \multicolumn{6}{|l|}{ MELIACEAE* } \\
\hline Cedrela odorata L. (cedro, cedro-branco) & $\mathrm{Av}$ & $\mathrm{N}$ & ef & - & \\
\hline \multicolumn{6}{|l|}{ MORACEAE* } \\
\hline Artocarpus communis Forst (fruta-pão) & Av & $\mathrm{C}$ & - & ae & \\
\hline A. integrifolia $\mathrm{L}$. (jaca) & Av & $\mathrm{C}$ & $\mathrm{abf}$ & acf & \\
\hline
\end{tabular}


Tabela 1 (continuação)

\begin{tabular}{|c|c|c|c|c|c|}
\hline Táxon & $\mathrm{H}$ & Fo & Ig & $\mathrm{Pa}$ & $\mathrm{Nc}$ \\
\hline \multicolumn{6}{|l|}{ MUSACEAE** } \\
\hline Musa cavendishii Lamb. (banana-anã) & $\mathrm{E}$ & $\mathrm{C}$ & $\mathrm{a}$ & - & \\
\hline M. paradisiaca L. (banana) & $\mathrm{E}$ & $\mathrm{C}$ & abe & ae & \\
\hline \multicolumn{6}{|l|}{ MYRISTICACEAE* } \\
\hline Sem determinação (urucuba) & Av & $\mathrm{N}$ & cef & $\mathrm{f}$ & \\
\hline \multicolumn{6}{|l|}{ MYRTACEAE* } \\
\hline Campomanesia dichotoma (Berg) Mattos (guabiraba) & $\mathrm{Ab}$ & $\mathrm{N}$ & $\mathrm{a}$ & - & 210 \\
\hline Eucalyptus citriodora Hook. (eucalipto) & Av & $\mathrm{C}$ & ce & - & 87,169 \\
\hline E. globulus Labill. (eucalipto-roxo) & Av & $\mathrm{C}$ & $\mathrm{cf}$ & - & \\
\hline Eugenia uniflora L. (pitanga) & Av & $\mathrm{C}$ & ae & ae & 38 \\
\hline Psidium guajava L. (goiaba) & Av & $\mathrm{C}$ & ace & ae & \\
\hline P. guineense Sw. (araçá) & Av & $\mathrm{N}$ & ae & acef & 30,104 \\
\hline Syzygium aromaticum Merr. \& L.M. Perry (cravo-da-índia) & Av & $\mathrm{O}$ & ae & - & \\
\hline S. jambolanum (Lam.) DC. (azeitona-roxa) & Av & $\mathrm{N}$ & ae & acef & 127,200 \\
\hline S. malaccensis L. (jambo) & Av & $\mathrm{C}$ & $\mathrm{a}$ & $\mathrm{a}$ & \\
\hline Sem determinação (mapirunga) & $\mathrm{Ab}$ & $\mathrm{N}$ & - & ae & \\
\hline Sem determinação (murta) & Av & $\mathrm{N}$ & $\mathrm{c}$ & - & 206 \\
\hline \multicolumn{6}{|l|}{ NYCTAGINACEAE* } \\
\hline Boerhaavia sp. (pega-pinto) & $\mathrm{E}$ & $\mathrm{N}$ & $\mathrm{e}$ & e & \\
\hline Boungaivillea glabra Choysi (arvoredo) & Av & $\mathrm{C}$ & - & - & 185 \\
\hline Guapira sp. (João-mole) & Av & $\mathrm{N}$ & $\mathrm{g}$ & $\mathrm{f}$ & \\
\hline Myrabilis jalapa L. (maravilha) & $\mathrm{E}$ & $\mathrm{C}$ & $\mathrm{g}$ & $\mathrm{g}$ & \\
\hline \multicolumn{6}{|l|}{ OCHNACEAE* } \\
\hline Ouratea hexasperma (A.St.-Hil.) Baill. (batiputá) & Av & $\mathrm{N}$ & $\mathrm{e}$ & - & 182 \\
\hline \multicolumn{6}{|l|}{ OLACACEAE* } \\
\hline Ximenia americana L. (ameixa-do-mato) & Av & $\mathrm{N}$ & ae & ae & 204 \\
\hline \multicolumn{6}{|l|}{ OXALIDACEAE* } \\
\hline Averrhoa carambola L. (carambola) & Av & $\mathrm{C}$ & ae & ae & 80 \\
\hline \multicolumn{6}{|l|}{ PASSIFLORACEAE* } \\
\hline Passiflora edulis Sims (maracujá) & $\mathrm{L}$ & $\mathrm{C}$ & $\mathrm{a}$ & a & \\
\hline P. quadrangularis L. (maracujá-açu) & $\mathrm{L}$ & $\mathrm{N}$ & ae & - & 239 \\
\hline Sem determinação (maracujá-mochila) & $\mathrm{L}$ & $\mathrm{N}$ & $\mathrm{e}$ & - & \\
\hline \multicolumn{6}{|l|}{ PIPERACEAE* } \\
\hline $\begin{array}{l}\text { Peperomia pellucida Humb., Bonplan \& Kunth. (língua-de- } \\
\text { sapo, mão-de-sapo) }\end{array}$ & $\mathrm{E}$ & $\mathrm{N}$ & $\mathrm{e}$ & e & 29 \\
\hline Piper marginatum Jacq. (malvaísco) & $\mathrm{E}$ & $\mathrm{N}$ & - & eg & 59,220 \\
\hline \multicolumn{6}{|l|}{ POACEAE** } \\
\hline Brachiaria mutica (Forssk.) Stapf (capim-de-planta) & $\mathrm{E}$ & $\mathrm{C}$ & - & e & \\
\hline Cymbopogon citratus Stapf (capim-santo) & $\mathrm{E}$ & $\mathrm{C}$ & ae & ae & 95 \\
\hline Dendrocalamus giganteus Munro (bambu) & $\mathrm{E}$ & I & $\mathrm{e}$ & $\mathrm{g}$ & 33 \\
\hline Imperata brasiliensis Trin. (sapé) & $\mathrm{E}$ & $\mathrm{N}$ & ce & - & 68 \\
\hline Lasiacis divaricata (L.) Hitchc. (taquari) & $\mathrm{E}$ & $\mathrm{N}$ & $\mathrm{f}$ & - & 27 \\
\hline Oryza sativa L. (arroz) & $\mathrm{E}$ & $\mathrm{O}$ & $\mathrm{a}$ & a & \\
\hline Pennisetum purpureum Schum. (capim-elefante) & $\mathrm{E}$ & $\mathrm{C}$ & $\mathrm{g}$ & - & \\
\hline Phalaris canariensis L. (alpiste) & $\mathrm{E}$ & $\mathrm{O}$ & - & $\mathrm{e}$ & \\
\hline Saccharum officinarum L. (cana-de-açúcar) & $\mathrm{E}$ & $\mathrm{C}$ & ag & $\mathrm{ab}$ & \\
\hline Zea mays L. (milho) & $\mathrm{E}$ & $\mathrm{C}$ & abd & $\mathrm{ad}$ & \\
\hline \multicolumn{6}{|l|}{ RHAMNACEAE* } \\
\hline Ziziphus joazeiro Mart. (juá) & Av & $\mathrm{N}$ & $\mathrm{e}$ & e & 126 \\
\hline \multicolumn{6}{|l|}{ ROSACEAE* } \\
\hline Pyrus malus L. (maçã) & Av & $\mathrm{O}$ & $\mathrm{a}$ & a & \\
\hline Rosa sp. 1 (rosa) & $\mathrm{E}$ & $\mathrm{C}$ & $\mathrm{g}$ & $\mathrm{g}$ & \\
\hline Rosa sp. 2 (rosa-amélia) & $\mathrm{E}$ & $\mathrm{C}$ & - & $\mathrm{g}$ & \\
\hline Rosa sp. 3 (rosa-branca) & $\mathrm{E}$ & $\mathrm{C}$ & $\mathrm{d}$ & $\mathrm{g}$ & \\
\hline Rosa sp. 4 (rosa-bugaí) & $\mathrm{E}$ & $\mathrm{C}$ & - & $\mathrm{g}$ & \\
\hline Rosa sp. 5 (rosa-cacho) & $\mathrm{E}$ & $\mathrm{C}$ & - & $\mathrm{g}$ & \\
\hline Rosa sp. 6 (rosa-menino) & $\mathrm{E}$ & $\mathrm{C}$ & - & $\mathrm{g}$ & \\
\hline Rosa sp. 7 (rosa-mesquita) & $\mathrm{E}$ & $\mathrm{C}$ & $\mathrm{e}$ & $\mathrm{g}$ & 222 \\
\hline
\end{tabular}


Tabela 1 (continuação)

\begin{tabular}{|c|c|c|c|c|c|}
\hline Táxon & $\mathrm{H}$ & Fo & $\operatorname{Ig}$ & $\mathrm{Pa}$ & $\mathrm{Nc}$ \\
\hline \multicolumn{6}{|l|}{ RUBIACEAE* } \\
\hline Borreria verticillata $\mathrm{G}$. Meyer (vassourinha-de-botão) & $\mathrm{E}$ & $\mathrm{N}$ & def & e & 31,99 \\
\hline Genipa americana L. (jenipapo) & Av & $\mathrm{N}$ & acf & ae & \\
\hline Guettarda platypoda DC. (angélica) & $\mathrm{Ab}$ & $\mathrm{N}$ & - & $\mathrm{e}$ & 61 \\
\hline Mussaendra alicia Hort (samumenha) & $\mathrm{E}$ & $\mathrm{C}$ & - & g & 214 \\
\hline Palicourea crocea (Sw.) Roem. \& Schult. (erva-de-rato) & $\mathrm{Ab}$ & $\mathrm{N}$ & $\mathrm{g}$ & - & 242 \\
\hline \multicolumn{6}{|l|}{ RUTACEAE* } \\
\hline Citrus limeta Risso (lima) & Av & $\mathrm{O}$ & $\mathrm{a}$ & - & \\
\hline C. limonia Osbeck (limão) & $\mathrm{Av}$ & $\mathrm{C}$ & $\mathrm{a}$ & - & \\
\hline C. sinensis (L.) Osbeck (laranja) & Av & $\mathrm{C}$ & abe & aef & \\
\hline Citrus sp. (mimo-do-céu) & Av & $\mathrm{C}$ & - & e & \\
\hline Fagara sp. (limãozinho) & Av & $\mathrm{N}$ & $\mathrm{f}$ & - & 201 \\
\hline Ruta graveolens L. (arruda) & $\mathrm{E}$ & $\mathrm{C}$ & de & $\operatorname{deg}$ & \\
\hline \multicolumn{6}{|l|}{ SAPINDACEAE* } \\
\hline Allophylus edulis (A.St.-Hil.) Mattos (guaxuma) & $\mathrm{Ab}$ & $\mathrm{N}$ & $\mathrm{f}$ & - & \\
\hline Cardiospermum halicacabum L. (cipó-de-vaqueiro) & $\mathrm{L}$ & $\mathrm{C}$ & $\mathrm{e}$ & - & 199 \\
\hline Cupania sp. (cabatan) & Av & $\mathrm{N}$ & $\mathrm{cf}$ & $\mathrm{cf}$ & 256 \\
\hline Paullinia elegans Cambess. (mata-fome) & $\mathrm{L}$ & $\mathrm{N}$ & $\mathrm{a}$ & - & 117 \\
\hline Serjania paucidentata DC. (trepadeira) & $\mathrm{L}$ & $\mathrm{N}$ & $\mathrm{c}$ & - & 212 \\
\hline Tallisia esculenta (A.St.-Hil.) Radlk. (pitomba) & Av & $\mathrm{C}$ & $\mathrm{a}$ & $\mathrm{a}$ & 142 \\
\hline Sem determinação (quiri) & Av & $\mathrm{N}$ & $\mathrm{cf}$ & $\mathrm{cf}$ & \\
\hline \multicolumn{6}{|l|}{ SAPOTACEAE* } \\
\hline Achras zapota (L.) Royen (sapoti) & Av & $\mathrm{C}$ & $\mathrm{a}$ & a & 143 \\
\hline Sideroxylon obtusifolium T.D. Penn. (quixaba) & Av & $\mathrm{O}$ & $\mathrm{e}$ & e & \\
\hline Manilkara sp. (maçaranduba) & Av & $\mathrm{N}$ & $\mathrm{cf}$ & - & \\
\hline Pradosia sp. (buranhém) & $\mathrm{Av}$ & $\mathrm{N}$ & $\mathrm{e}$ & - & \\
\hline Sem determinação (abiu) & Av & $\mathrm{N}$ & $\mathrm{a}$ & - & \\
\hline \multicolumn{6}{|l|}{ SCROPHULARIACEAE* } \\
\hline Scoparia dulcis L. (vassourinha-de-rezar) & $\mathrm{E}$ & $\mathrm{N}$ & $\mathrm{d}$ & $\mathrm{d}$ & 48,83 \\
\hline \multicolumn{6}{|l|}{ SIMAROUBACEAE* } \\
\hline Simarouba amara Aubl. (praíba) & Av & $\mathrm{N}$ & $\mathrm{cf}$ & $\mathrm{c}$ & 94 \\
\hline \multicolumn{6}{|l|}{ SMILACACEAE** } \\
\hline Smilax sp. (japecanga) & $\mathrm{L}$ & $\mathrm{N}$ & $\mathrm{e}$ & e & 93,192 \\
\hline \multicolumn{6}{|l|}{ SOLANACEAE* } \\
\hline Capsicum annuum L. (pimentão) & $\mathrm{E}$ & $\mathrm{O}$ & ag & a & \\
\hline Lycopersicum cesariforme Dun (tomate-miudinha) & $\mathrm{E}$ & $\mathrm{N}$ & $\mathrm{e}$ & - & 259 \\
\hline L. esculentum Mill. (tomate) & $\mathrm{E}$ & $\mathrm{C}$ & $\mathrm{a}$ & a & \\
\hline Solanum agrarium Sendtn. (gogóia) & $\mathrm{E}$ & $\mathrm{N}$ & - & a & 193 \\
\hline Solanum americanum Mill. (erva-moura) & $\mathrm{E}$ & $\mathrm{N}$ & $\mathrm{e}$ & $\mathrm{e}$ & 65,101 \\
\hline S. paniculatum L. (jurubeba) & $\mathrm{E}$ & $\mathrm{N}$ & - & e & 105,136 \\
\hline S. tuberosum L. (batatinha) & $\mathrm{E}$ & $\mathrm{C}$ & ae & - & \\
\hline \multicolumn{6}{|l|}{ STERCULIACEAE* } \\
\hline Guazuma ulmifolia L. (mutamba) & Av & $\mathrm{N}$ & - & eg & 42,132 \\
\hline Theobroma cacao L. (cacau) & Av & $\mathrm{C}$ & $\mathrm{a}$ & - & \\
\hline \multicolumn{6}{|l|}{ TILIACEAE* } \\
\hline Apeiba tiborbou Aubl. (jangada) & $\mathrm{Av}$ & $\mathrm{N}$ & $\mathrm{f}$ & - & \\
\hline Luehea ochrophylla Mart. (pereiro) & $\mathrm{Ab}$ & $\mathrm{N}$ & - & $\mathrm{cf}$ & 51 \\
\hline Triunfetta abutiloides A.St.-Hil. (carrapicho). & $\mathrm{Ab}$ & $\mathrm{N}$ & $\mathrm{f}$ & - & 115 \\
\hline \multicolumn{6}{|l|}{ TURNERACEAE } \\
\hline Turnera ulmifolia L. (chanana) & $\mathrm{E}$ & $\mathrm{N}$ & - & e & \\
\hline \multicolumn{6}{|l|}{ URTICACEAE } \\
\hline Pilea microphylla (zezinho) & $\mathrm{E}$ & $\mathrm{C}$ & - & g & \\
\hline \multicolumn{6}{|l|}{ VERBENACEAE } \\
\hline Lantana camara L. (chumbinho) & $\mathrm{Ab}$ & $\mathrm{N}$ & $\mathrm{e}$ & - & 28 \\
\hline Lippia sp. (erva-cidreira) & $\mathrm{E}$ & $\mathrm{I}$ & $\mathrm{e}$ & $\mathrm{e}$ & 66 \\
\hline Stachytarpheta elatior Schrad. (mocotó) & $\mathrm{E}$ & $\mathrm{N}$ & $\mathrm{e}$ & - & 254 \\
\hline Vitex agnus-castus L. (liamba) & $\mathrm{E}$ & $\mathrm{C}$ & $\mathrm{e}$ & - & 81 \\
\hline
\end{tabular}


Tabela 1 (continuação)

\begin{tabular}{|c|c|c|c|c|c|}
\hline Táxon & $\mathrm{H}$ & Fo & Ig & $\mathrm{Pa}$ & $\mathrm{Nc}$ \\
\hline \multicolumn{6}{|l|}{ VIOLACEAE* } \\
\hline Hybanthus calceolaria (L.) Schulze-Menz (pepaconha) & $\mathrm{E}$ & $\mathrm{N}$ & e & e & 174,202 \\
\hline \multicolumn{6}{|l|}{ VITACEAE* } \\
\hline Cissus verticillata (L.) Nicolson \& Jarvis (ansiline) & $\mathrm{E}$ & $\mathrm{C}$ & e & - & 235 \\
\hline Leea sp. (café) & $\mathrm{Ab}$ & $\mathrm{C}$ & $\operatorname{deg}$ & $\mathrm{g}$ & 69 \\
\hline Vitis vinifera $\mathrm{L}$. (uva) & $\mathrm{L}$ & $\mathrm{O}$ & $\mathrm{a}$ & a & \\
\hline \multicolumn{6}{|l|}{ ZINGIBERACEAE** } \\
\hline Alpinia zerumbet (Pers.) Burt ex R.M. Sm. (colônia) & $\mathrm{E}$ & $\mathrm{C}$ & de & de & 187 \\
\hline Costus sp. 1 (cana-de-macaco) & $\mathrm{E}$ & $\mathrm{C}$ & $\mathrm{e}$ & - & 228 \\
\hline Costus sp. 2 & $\mathrm{E}$ & $\mathrm{C}$ & - & e & 157 \\
\hline \multicolumn{6}{|l|}{ INDETERMINADAS } \\
\hline Alfinete & $\mathrm{E}$ & $\mathrm{C}$ & $\mathrm{g}$ & g & \\
\hline Batata-contra-erva & $\mathrm{E}$ & $\mathrm{N}$ & e & - & \\
\hline Bredo-alemão & $\mathrm{L}$ & $\mathrm{C}$ & a & - & \\
\hline Caeté & $\mathrm{E}$ & $\mathrm{N}$ & $\mathrm{c}$ & - & \\
\hline Capitão/capitão-preto & $\mathrm{E}$ & $\mathrm{N}$ & ae & - & \\
\hline Casquinho/cabo-de-machado & Av & $\mathrm{N}$ & $\mathrm{f}$ & - & \\
\hline Chambá-do-sertão & $\mathrm{E}$ & $\mathrm{C}$ & e & - & \\
\hline Coça-coça-branca & $\mathrm{E}$ & $\mathrm{N}$ & - & $\mathrm{e}$ & \\
\hline Cueira, pau-falha & Av & $\mathrm{N}$ & $\mathrm{cf}$ & - & \\
\hline Dor-de-cabeça & $\mathrm{Ab}$ & $\mathrm{N}$ & $\mathrm{a}$ & - & \\
\hline Espinheiro & Av & $\mathrm{N}$ & - & $\mathrm{f}$ & \\
\hline Esquentai & $\mathrm{E}$ & $\mathrm{N}$ & $\mathrm{e}$ & - & \\
\hline Fava-de-cheiro & $\mathrm{L}$ & $\mathrm{N}$ & $\mathrm{a}$ & - & \\
\hline Gindiobra & $\mathrm{E}$ & $\mathrm{N}$ & $\mathrm{e}$ & - & \\
\hline Grão-de-galo & Av & $\mathrm{N}$ & - & $\mathrm{a}$ & \\
\hline Jamaica & $\mathrm{E}$ & $\mathrm{C}$ & - & $\mathrm{g}$ & \\
\hline João-mole & Av & $\mathrm{N}$ & e & - & \\
\hline Leitero & Av & $\mathrm{N}$ & $\mathrm{c}$ & - & \\
\hline Merassilina & $\mathrm{E}$ & $\mathrm{C}$ & $\mathrm{e}$ & - & \\
\hline Novalgina & $\mathrm{E}$ & $\mathrm{C}$ & $\mathrm{e}$ & - & \\
\hline Pena-branca & $\mathrm{E}$ & $\mathrm{C}$ & - & g & \\
\hline Ronçara & $\mathrm{E}$ & $\mathrm{N}$ & $\mathrm{e}$ & - & \\
\hline Sete-casco & Av & $\mathrm{N}$ & - & $\mathrm{cf}$ & \\
\hline Tapacu & Av & $\mathrm{N}$ & - & $\mathrm{a}$ & \\
\hline Urinana & $\mathrm{E}$ & $\mathrm{N}$ & $\mathrm{e}$ & - & \\
\hline
\end{tabular}

no sul da Bahia, também relata que $58 \%$ das espécies de uso terapêutico na região são cultivadas. Na Baía de Sepetiba (RJ), Figueiredo et al. (1997) também relataram situação semelhante no que se refere às plantas medicinais.

As duas comunidades apresentaram em comum 173 espécies úteis, diferindo, em alguns casos, quanto à categoria de uso, sendo a maioria cultivada $(54,9 \%)$. Aplicando-se o Quociente de Similaridade de Sørensen, registrou-se o valor de $\mathrm{Qs}_{\mathrm{IgPa}}=0,69$, indicando que as comunidades efetivamente se assemelham quanto ao conhecimento de espécies úteis.

Alimentação - Nesta categoria foram incluídas 115 espécies (Tab. 1), com uma razoável diversidade em cada comunidade ( $\mathrm{Ig}=35,1 \% ; \mathrm{Pa}=36,4 \%)$, correspondendo a plantas usadas apenas na alimentação humana, em suas diversas formas de uso, predominantemente cultivadas, como as frutíferas (Cocos nucifera - coco; Mangifera indica - manga; Musa paradisiaca - banana); as espécies com raízes e tubérculos comestíveis, como Ipomoea batatas (batata doce), Manihot esculenta (macaxeira/ mandioca) e Solanum tuberosum (batatinha); as verduras, entre as quais destacam-se Coriandrum sativum (coentro), Daucus carota (cenoura) e Hibiscus esculentus (quiabo); e as fornecedoras de grãos, como Phaseolus vulgaris (feijão) e Zea mays (milho); e algumas nativas, como Hymenaea martiana 
(jatobá) e Psidium guineense (araçá). Além disso, foram também registradas algumas espécies exóticas adquiridas no comércio, como Brassica oleracea (couve/repolho) e Pyrus malus (maçã).

O cultivo de roças, pequenas hortas e pomares mostrou-se uma atividade essencial para a subsistência das comunidades estudadas, colocando num segundo plano a coleta de alimentos nas áreas remanescentes de vegetação próximas às mesmas. No entanto, a atividade agrícola vem sendo gradativamente suprimida, por causa de perda de acesso à terra para plantio pelos moradores. Na Usina São José (Ig) as famílias foram retiradas de seus sítios e impedidas de praticar a lavoura, passando a adquirir através da compra a maior parte do alimento necessário para sua subsistência. Mesmo assim, as espécies cultivadas ainda são as principais fontes de alimentação nas comunidades, com $59,00 \%(\mathrm{Ig})$ e $64,10 \%(\mathrm{~Pa})$. Desse modo, as espécies nativas representam atualmente uma fonte secundária de recursos alimentares, fornecendo frutos e sementes, com baixo número de espécies citadas em cada comunidade $(\mathrm{Ig}=30,0 \% ; \mathrm{Pa}=25,6 \%)$. Esses valores podem ser comparados com os obtidos por Sales \& Lima (1985) que, trabalhando com a flora da Caatinga, em Soledade, Paraíba, registraram que 35\% das espécies são usadas na alimentação. Outros estudos, realizados na Região Amazônica, com espécies arbóreas nativas, também registraram uma baixa diversidade para uso na alimentação, com 37,6\% (Baleé 1986), 25,4\% (Baleé 1987) e 28,2\% (Pinedo-Vasquez et al. 1990), demonstrando que é secundário o uso de espécies nativas na alimentação, mesmo em áreas com ampla cobertura vegetal.

A maioria das espécies citadas como alimento em Jaguarana (Pa), apresenta hábito arbóreo e arbustivo $(55,13 \%)$. Já na Usina São José (Ig), foi registrada a mesma ocorrência de uso entre árvores/arbustos e ervas/lianas $(50,00 \%)$, decorrente da maior atividade agrícola (lavoura) entre os moradores desta comunidade. O elevado índice de similaridade encontrado para esta categoria de uso $\left(\mathrm{Qs}_{\mathrm{IgPa}}=0,71\right)$, demonstra que, apesar das diferentes origens e tipos de atividade, as duas comunidades utilizam muitas espécies em comum na alimentação, devido encontrarem-se localizadas numa mesma região fitogeográfica.

Comércio - Nesta categoria foram incluídas 28 espécies, provenientes de duas fontes distintas (Tab. 1). Na primeira enquadram-se aquelas coletadas nas áreas de matas vizinhas a cada comunidade, com poucas espécies citadas, como Acrocomia intumescens (macaíba), cujos frutos são coletados e vendidos. Na segunda, com maioria de citações, registram-se as plantas cultivadas, como Ipomoea batatas (batata doce), Manihot esculenta (macaxeira/ mandioca) e Solanum tuberosum (batatinha), cujas raízes e tubérculos são comercializados. Em ambos, tanto o número de espécies citadas em cada comunidade foi reduzido ( $\mathrm{Ig}=8,1 \% ; \mathrm{Pa}=4,7 \%$ ), quanto o número de citações por espécie, indicando que os vegetais não apresentam importância relevante na atividade de comércio nas comunidades, servindo apenas como um complemento de renda para as famílias. A maioria das espécies citadas são ervas e lianas, com percentuais de 56,5\% (Ig) e 70,0\% (Pa), mas com representatividade de árvores e arbustos cujos frutos são comercializáveis. Por ter revelado um perfil urbano, a comunidade de Jaguarana $(\mathrm{Pa})$ apresentou baixa similaridade de plantas utilizadas no comércio em relação à Usina São José $\left(\mathrm{Qs}_{\mathrm{IgPa}}=0,30\right)$, devido à reduzida citação de espécies enquadradas nesta categoria de uso.

Construção - Foram incluídas nesta categoria 55 espécies, nas duas áreas de estudo (Tab. 1). Todas são plantas empregadas pelas comunidades para construção de habitações e locais de trabalho, como barracas e currais, fornecendo madeira para linhas, caibros, ripas e tábuas; materiais (palha de coqueiro, capim seco etc) usados para cobertura de telhados; lianas utilizadas para amarrar as estruturas. Neste grupo enquadram-se, majoritariamente, espécies nativas $(87,3 \%)$, e de porte arbóreo $(94,5 \%)$, como Bowdichia virgilioides (sucupira), Eschweilera ovatta (embiriba) e Tabebuia avellanedae (paud'arco-roxo); apenas algumas são cultivadas, entre as quais se incluem Artocarpus integrifolia (jaca) e Genipa americana (jenipapo).

Foi registrado um reduzido número de espécies para esta categoria de uso, em relação ao total de espécies citadas nas comunidades estudadas $(\mathrm{Ig}=15,8 \% ; \mathrm{Pa}=11,3 \%)$. Esses baixos valores estariam associados à dificuldade dos moradores, em cada comunidade, de usarem plantas madeireiras existentes nas matas, em virtude da fiscalização dos órgãos públicos de proteção ao meio ambiente, impedindo o corte; o fato das áreas onde podem ser encontradas essas espécies serem propriedades privadas, dificulta mais ainda o acesso às mesmas. Outras regiões, com maior disponibilidade de cobertura vegetal, podem apresentar uma maior diversidade de espécies usadas em construções, como foi registrado 
por Baleé $(1986 ; 1987)$ no Maranhão $(23,9 \%)$ e no Pará (37,7\%), por Pinedo-Vasquez et al. (1990) na Amazônia Peruana (31,3\%) e também por V.A. Silva (dados não publicados) em Pernambuco (32,1\%). Ao se comparar o conjunto de espécies utilizadas para construção, verifica-se que apresentam características próprias para cada comunidade, com baixo índice de similaridade $\left(\mathrm{Qs}_{\mathrm{IgPa}}=0,35\right)$. Esta diferença pode ser decorrente da maior citação de espécies cultivadas em Igarassu.

Mágica - Foram incluídas nesta categoria de uso 28 espécies usadas no tratamento de problemas espirituais, como amuletos de sorte e para trazer bons presságios e proteção, além de outros significados (Tab. 1). Elas foram indicadas sob várias formas de uso, como banhos, defumadores, benzeduras e rezas, usando-se toda a planta ou parte dela. Várias espécies foram citadas nas duas áreas, incluindo Jatropha gossypiifolia (pinhão-roxo), Ruta graveolens (arruda), espécies cultivadas e Scoparia dulcis (vassourinha-de-rezar), uma planta ruderal, sendo a maioria de porte herbáceo (64,3\%), indicando a importância mínima da vegetação nativa da Mata Atlântica na visão mística das comunidades estudadas, já que as duas primeiras resultam da influência africana.

As comunidades da Usina São José (Ig) e de Jaguarana (Pa) apresentaram um conjunto semelhante de espécies citadas $\left(\mathrm{Qs}_{\mathrm{IgPa}}=0,53\right)$, ambas tendo apresentado quase o mesmo número de espécies ( $\mathrm{Ig}=18$ sp.; $\mathrm{Pa}=20 \mathrm{sp}$.), além dos mesmos valores relativos à origem das espécies utilizadas, com predominância de cultivadas $(57,1 \%)$, mas boa representatividade de espécies nativas e introduzidas (42,9\%). Muitas destas espécies foram registradas como mais conhecidas e utilizadas nos estudos de Albuquerque \& Chiappeta (1996/1997) enfocando o uso das plantas em rituais afro-brasileiros no Recife, Pernambuco.

Medicinal - Esta categoria foi a que apresentou maior número de espécies em todas as comunidades estudadas, com 48,8\% (Ig) e 44,4\% (Pa), correspondendo a quase metade do total das plantas citadas pelos informantes (169 sp.). Em vários trabalhos realizados no Brasil, esta categoria também aparece entre as mais representativas, em relação ao número de espécies citadas, seja em comunidades urbanas, rurais ou aborígenes (Anderson \& Posey 1985; Sales \& Lima 1985; Balée 1986; 1987; Kayner \& Duryea 1992; Begossi et al. 1993; Figueiredo et al. 1993; Silva 1997; M.F.S. Almeida, dados não publicados).
Foram registradas plantas utilizadas no tratamento de doenças respiratórias, como Acanthospermum hispidum (espinho-de-cigano), Chenopodium ambrosioides (mastruz) e Plectranthus amboinicus (hortelã graúda); problemas no aparelho digestivo, entre as quais Lippia sp. (erva-cidreira), Plectranthus barbatus (boldo) e Psidium guajava (goiaba); como antifebris foram citadas Alpinia zerumbet (colônia) e Pfaffia glomerata (acônito), entre outras; para tratamento de problemas renais citaram-se Averrhoa carambola (carambola), Cereus jamacaru (cardeiro) e Phyllanthus amarus (quebra-pedra); com atividade antiinflamatória e cicatrizante, Anacardium occidentale (caju-roxo), Pithecellobium cochliocarpum (barbatenon) e Schinus terebintifolius (aroeira), entre outras indicações.

Mesmo com uma grande diversidade de plantas citadas nesta categoria (Tab. 1), as comunidades apresentaram-se semelhantes no conjunto de espécies usadas com fins terapêuticos $\left(\mathrm{Qs}_{\mathrm{IgPa}}=0,56\right)$, bastante influenciadas pelo percentual de espécies nativas indicadas (46,7\%). Apesar da acentuada presença de espécies nativas, e concordando com o observado por Branch \& Silva (1983) e Victor \& Andrade (1991), as plantas de uso terapêutico mais citadas nas duas comunidades corresponderam àquelas registradas em vários estudos realizados em diferentes regiões do Brasil, em comunidades rurais e urbanas, evidenciando a perda de conhecimento sobre espécies medicinais nativas (Mariz et al. 1986; Amorozo \& Gély 1988; Pereira et al. 1988; Grandi et al. 1989; Rizzo et al. 1990). Da mesma forma que nas comunidades caiçaras estudadas por Hanazaki et al. (2000), entre as plantas medicinais destacaram-se em número as exóticas cultivadas, de origem africana e européia, Sambucus australis Cham. \& Schlecht, Aeolanthus suaveolens Mart. ex Spreng., e Ocimum gratissimum L. Todavia espécies nativas, como por exemplo Cedrela fissilis Vell., Eugenia uniflora L. e Bidens pilosa L. parecem ter grande importância nas duas comunidades, como sugerem os percentuais de citações obtidos.

As ervas apresentaram-se mais freqüentemente utilizadas para fins terapêuticos, com 53,5\% (Ig) e $54,2 \%(\mathrm{~Pa})$, sendo pouco representativas as arbóreas e arbustivas, em concordância com o observado pela maioria dos autores, como Amorozo \& Gély (1988) e Voeks (1996).

Tecnológica - Nesta categoria foram incluídas 70 espécies, utilizadas na confecção de ferramentas, equipamentos, móveis e utensílios domésticos, como Bowdichia virgilioides (sucupira), Guatteria 
australis (embira-preta) e Xylopia frutescens (embira-vermelha); no fornecimento de lenha e carvão, entre elas Byrsonima sericea (murici) e Tapirira guianensis (cupiúba) e outras usadas na realização de atividades específicas (Tab. 1). São também poucas as espécies enquadradas como de uso tecnológico nas diferentes comunidades ( $\mathrm{Ig}=19,3 \% ; \mathrm{Pa}=15,4 \%)$, com maior representatividade de espécies nativas $(87,1 \%)$ e arbóreo/arbustivas (90,0\%).

As comunidades não se apresentaram semelhantes em relação às plantas citadas como de uso tecnológico $\left(\mathrm{Qs}_{\mathrm{IgPa}}=0,41\right)$; a da Usina São José (Ig), que apresentou maior número de espécies (55), caracteriza-se como um conjunto de comunidades rurais situadas distante de centros urbanos, o que as torna mais dependentes dos recursos vegetais encontrados na região, para confecção de ferramentas e utensílios necessários à subsistência, além da obtenção de combustível (lenha, carvão). Em Jaguarana $(\mathrm{Pa})$, onde ocorreu o menor número de espécies citadas (33), com um perfil urbano e localizando-se próxima ao centro da Cidade do Paulista, existem facilidades para seus moradores adquirirem ferramentas, utensílios, móveis e madeira no comércio local, além da maior disponibilidade de fontes de energia (eletricidade, gás de cozinha, combustíveis), não necessitando assim, de explorar intensivamente os recursos vegetais locais.

Outros - Esta categoria, que inclui 56 espécies, é uma miscelânea de diversos usos indicados para as plantas que não puderam ser incluídos nas anteriores (Tab. 1). Foram registradas espécies de uso cosmético, como Guazuma ulmifolia (mutamba) e Zizyphus joazeiro (juá); plantas cujas flores são usadas em ornamentação, como Catharanthus roseos (boa-noite), Rosa sp. (rosa) e Hibiscus rosa-sinensis (papoula), cultivadas em jardins residenciais; outras espécies ornamentais pelas folhas, utilizadas como adornos em jarros, caqueiras e enfeites, como Dieffenbachia picta (comigo-ninguém-pode) e Lycopodiella cernua (unha de gato); árvores utilizadas para arborização de locais públicos, entre elas Caesalpinia echinata (pau-brasil). A similaridade entre as comunidades foi baixa $\left(\mathrm{Qs}_{\mathrm{IgPa}}=0,47\right)$, por causa da diversidade de fins em que são utilizadas as plantas enquadradas nesta categoria, a maioria cultivada (67,9\%).

Tomando-se os dados obtidos em conjunto, constata-se que a maior diversidade de espécies úteis encontra-se no grupo das plantas medicinais, seguidas das alimentícias. As comunidades estudadas subutilizam a vegetação nativa como fonte de recursos alimentares. $\mathrm{O}$ fato de ocorrer um equilíbrio em relação aos hábitos arbóreo e herbáceo das plantas utilizadas como alimento, reflete a existência de muitos pomares nas comunidades. No grupo das espécies medicinais, embora se observe certa predominância de ervas, constata-se a tendência a serem utilizadas com a mesma freqüência espécies nativas e cultivadas, com boa representatividade de árvores, arbustos e lianas nativas, indicando que a vegetação local é uma fonte importante de recursos medicinais para as comunidades estudadas.

O fato das categorias de uso construção e tecnologia apresentarem os maiores índices de espécies nativas úteis para as comunidades, demonstra que a vegetação local é fonte essencial de recursos madeireiros, utilizados na construção de casas, barracas, cercas, na fabricação de ferramentas, utensílios domésticos e instrumentos diversos, e também como combustível (lenha e carvão). Assim, um dos principais recursos retirados das matas vizinhas às comunidades estudadas é a madeira, cuja exploração desordenada diminui os já pequenos fragmentos florestais envolvidos, contribuindo para a sua degradação.

De modo geral, as plantas de valor comercial são mais importantes na subsistência das comunidades, do que para a geração de renda, embora na Usina São José (Ig), onde a atividade agrária foi mais relevante em épocas passadas, o comércio de raízes, frutas, legumes e cereais tenha tido um papel importante no dia-a-dia dessas comunidades. A baixa diversidade de plantas usadas como mágicas e místicas, dentre as quais observa-se a predominância de espécies cultivadas, demonstra que a vegetação nativa apresenta pouca relevância na visão cosmológica das comunidades estudadas. A maior freqüência de espécies cultivadas e de hábito herbáceo evidencia que as comunidades pouco utilizam a vegetação nativa para fins comerciais ou em suas rezas e crenças.

Localizadas numa mesma região fitogeográfica, as comunidades estudadas compartilham algumas características e apresentam outras próprias, decorrentes tanto das principais atividades que nelas exercem seus moradores, como dos recursos naturais à sua disposição. As comunidades da Usina São José (Ig), situadas numa área rural, formadas principalmente por trabalhadores agrícolas que atuam na lavoura canavieira e cultivam roças e sítios, possuem uma maior diversidade de plantas úteis, tanto cultivadas quanto nativas, estas encontradas principalmente nas áreas de mata próximas. Jaguarana $(\mathrm{Pa})$, formada inicialmente por operários da indústria têxtil no 
Município de Paulista, mesmo sendo caracteristicamente uma vila urbana, apresenta uma razoável diversidade de plantas úteis, a maioria cultivada em jardins e pequenos sítios, mas com várias espécies nativas encontradas na mata vizinha à comunidade.

\section{Agradecimentos}

À Coordenadoria de Aperfeiçoamento de Pessoal de Nível Superior (CAPES), pela concessão de bolsa de estudos ao primeiro autor durante a realização deste trabalho. Ao Herbário IPA - Dárdano de Andrade Lima, pela utilização de suas instalações e equipamentos, durante a coleta e herborização do material botânico. Aos pesquisadores da Seção de Botânica e Ecologia da Empresa Pernambucana de Pesquisa Agropecuária (IPA), pela indispensável colaboração na identificação do material botânico e por valiosas sugestões.

\section{Referências bibliográficas}

Albuquerque, U.P. 1999. La importancia de los estudios etnobiológicos para establecimiento de estrategias de manejo y conservación en las florestas tropicales. Biotemas 12(1): 31-47.

Albuquerque, U.P. \& Chiappeta, A.A. 1996/1997. Levantamento das espécies vegetais empregadas nos cultos afro-brasileiros em Recife-PE. Biologica Brasilica 7: 9-22.

Amorozo, M.C.M. \& Gély, A. 1988. Uso de plantas medicinais por caboclos do baixo Amazonas, Barbacena, Pará, Brasil. Boletim do Museu Paraense Emílio Goeldi, Botânica 4(1): 47-131.

Anderson, A.B. \& Posey, D.A. 1985. Manejo de cerrado pelos índios Kayapó. Boletim do Museu Paraense Emílio Goeldi, Botânica 2(1): 77-98.

Andrade, M.C. 1988. Área do sistema canavieiro. Recife, SUDENE.

Baleé, W. 1986. Análise preliminar de inventário florestal e a Etnobotânica Ka'apor (Maranhão). Boletim do Museu Paraense Emílio Goeldi, Botânica 2(2): 141-167.

Baleé, W. 1987. A etnobotânica quantitativa dos índios Tembé (Rio Gurupi, Pará). Boletim do Museu Paraense Emílio Goeldi, Botânica 3(1): 29-47.

Begossi, A.; Leitão Filho, H.F. \& Richerson, P.J. 1993. Plant uses in a Brazilian coastal fishing community (Búzios Island). Journal of Ethnobiology 13(2): 233-256.

Branch, L.C. \& Silva, M.F. 1983. Folk medicine of Alter do Chão, Pará, Brazil. Acta Amazonica 13(5-6): 737-797.

Costa, E.M.P. 1982. Expansão urbana e organização espacial. Recife, Editora Universitária.

FIDEM - Fundação de Desenvolvimento da Região Metropolitana do Recife. 1987. Reservas Ecológicas. Série Desenvolvimento Urbano e Meio Ambiente. Recife, FIDEM.
FIDEM - Fundação de Desenvolvimento da Região Metropolitana do Recife. 1998. Perfis municipais. Recife, FIDEM.

Figueiredo, G.M.; Leitão Filho, H.F. \& Begossi, A. 1993. Ethnobotany of Atlantic Forest Coastal communities: Diversity of plant uses in Gamboa (Itacuruçá Island, Brazil). Human Ecology 21(4): 419-430.

Figueiredo, G.M.; Leitão Filho, H.F. \& Begossi, A. 1997. Ethnobotany of Atlantic Forest Coastal communities: II. Diversity of plant uses at Sepetiba Bay (SE Brazil). Human Ecology 21(4): 419-430.

Grandi, T.S.M.; Trindade, J.A.; Pinto, M.J.F.; Ferreira, L.L. \& Catella, A.C. 1989. Plantas medicinais de Minas Gerais, Brasil. Acta Botanica Brasilica, Suplemento 3(2): 185-224.

Hanazaki, N.; Tamashiro, J.Y.; Leitão Filho, H.F. \& Begossi, A. 2000. Diversity of plant uses in two Caiçara communities from the Atlantic Forest coast, Brazil.

Holmgren, P.R.; Holmgren, N.H. \& Barret, L.C. 1990. Index Herbariorum Part I. The herbaria of the World, ed 8. New York, New York Botanical Garden.

Kainer, K.A. \& Duryea, M.L. 1992. Tapping women's knowledge: plan resource use in extractive reserves, Acre, Brazil. Economic Botany 46(4): 408-425.

Mariz, G.; Lira, O.C.; Andrade, L.H.C.; Pires, M.G.M. \& Silva, S.I. 1986. Plantas utilizadas para cura de doenças na cidade do Recife-PE. Pp. 158-176. In: P. Scott (org.). Sistemas de cura: as alternativas do povo. Mestrado em Antropologia/UFPE. Recife.

Melo, M.L. 1978. Metropolização e subdesenvolvimento: o caso do Recife. Recife, Departamento de Ciências Geográficas - CFCH/UFPE.

Pereira, C.M.P.; Perazzolo, M.; Susin, U.L.N. \& Bersgesch, M. 1988. Levantamento preliminar dos vegetais medicamentosos existentes no Município de Rio Grande, RS. Acta Amazonica, Suplemento 18(1-2): 49-59.

Phillips, O.; Gentry, A.H.; Reynel, C.; Wilkin, P. \& GálvesDurand, B.C. 1994. Quantitative ethnobotany and Amazonian conservation. Conservation Biology 8(1): 225-248.

Pinedo-Vasquez, M.; Zarin, D.; Jipp, P. \& Chota-Inuma, J. 1990. Use-value of tree species in communal forest reserve in Northeast Peru. Conservation Biology 4(4): 405-416.

Prance, G.T.; Baleé, W.; Boom, B.M. \& Carneiro, R.L. 1987. Quantitative Ethnobotany and the case for conservation in Amazonia. Conservation Biology 1(4): 296-310.

Rizzo, J.A.; Monteiro, M.S.R. \& Bittencourt, C. 1990. Utilização de plantas medicinais em Goiânia. Pp. 691-714. In: Anais do XXXVI Congresso Nacional de Botânica. Curitiba, 1985. Brasília, Sociedade Botânica do Brasil.

Sales, M.F. \& Lima, M.J.A. 1985. Formas de uso da flora da Caatinga pelo assentamento da Microrregião de Soledade (PB). Pp. 165-184. In: Anais da VIII Reunião Nordestina de Botânica. Recife, 1984. Recife, Sociedade Botânica do Brasil - Seccional de Pernambuco. 
Sørensen, T. 1978. A method of establishing groups of equal amplitude in plant sociology based on similarity of species contents. In: R. McIntosh (ed.). Phytosociology. Pennsylvania, Dowdew, Hutchinson \& Ross, Inc.
Victor, P. \& Andrade, L.H.C. 1991. Flora medicinal: estudo comparativo entre dois municípios de Pernambuco. Biologica Brasilica 3(2): 179-200.

Voeks, R.A. 1996. Tropical forest healers and habitat preference. Economic Botany 50(4): 381-400. 